\title{
Conflict Triggers in International Business Negotiations
}

\author{
Stefanie Alexa Stadler
}

School of Humanities and Social Sciences, Nanyang Technological University, Singapore

Copyright $\mathrm{C} 2016$ by authors, all rights reserved. Authors agree that this article remains permanently open access under the terms of the Creative Commons Attribution License 4.0 international License.

\begin{abstract}
Conflict, is - arguably - one of the greatest inhibitors to successful interaction and desirable interactional outcomes. As international business people frequently work in new and/or unfamiliar cultural settings, they rely on social interactions that are almost invariably of an intercultural nature. As such, it is vital for them to learn to manage and navigate communication smoothly and successfully. However, intercultural communication is prone to misunderstandings and miscommunication based on different pragmatic norms and culture-specific communication practices. The consequences in such settings can be costly to the bottom line and irreparable to well-functioning business relationships. Recognizing and responding to potential interactional conflict in a timely and efficient manner is therefore paramount. In this paper, I focus on conflict triggers and conflict markers that indicate irritation, dissent or disagreement before conflict actually arises. A discourse analysis approach of video-recorded business interactions forms the basis for this discussion, in which I will demonstrate cultural differences in the approach to marking and indicating the onset of potential conflict and intercultural competencies conducive of resolving conflict in its early developmental stages.
\end{abstract}

Keyword Business Communication, Culture, Intercultural Competence, Discourse Analysis

\section{Introduction}

Communication is absolutely central to successful international business. Unfortunately, speaking the other party's language is not sufficient to guarantee successful interactions and much of the discrepancies in communication are due to pragmatic differences and cultural preferences for particular ways of using communication, with language functioning merely as a tool (Stadler, 2011). As interactional partners in business settings rarely completely align in their views and goals, managing business transactions is particularly prone to conversational conflict. The particularly high propensity for conflict in intercultural communication is documented in studies such as Spencer-Oatey and Xing (2008), who investigated business deals that went awry on the grounds of culture-based communication breakdown and led to considerable damage for inter-organizational relations. The tendency for intercultural contact to lead to more and more complex conflict situations is also documented by Elmer (1993) and Von Glinow et al. (2004). Conflict here is understood as interactional conflict that arises as a result of differences in opinions, disagreements on terms and conditions and a general lack of communicational alignment. Unfortunately, conflict is not always conducive to successful business transactions and it need not come in the shape of a complete fall-out between parties to aversely affect communication and relationships.

Since disagreements and differences in points of view are integral to negotiation, it is impossible to avoid negotiations on differences in opinions and terms. Richmond and McCroskey (2010) see disagreement as a normal and essential aspect of everyday conversation, while they see conflict as an escalated form of personalized disagreement, stating that "when disagreement is personalized it becomes verbal aggression and the interaction becomes conflict" ( $p$. 361).

It is necessary at this point to distinguish between different types of conflict. Researchers have started to make a distinction between what is commonly referred to as task based conflict and relationship conflict (cf. e.g. DeDreu \& Weingart, 2003). While task based conflict (of a small to moderate amount and when well managed) can actually foster discussion, multiple viewpoints and hence also creativity, productivity and effective decision-making (Simons \& Peterson, 2000; Tjosvold, 2008), the same cannot be said for relational conflict. Relationship conflict is never conducive. Relationship conflict is associated with poor decision-making, high stress levels and anxiety, and low group satisfaction and commitment (Simons \& Peterson, 2000). An escalation from disagreement to conflict should therefore be avoided at all cost, as relationships rarely fully recover from fall-outs. As Friedman and Currall (2003) put it: "One reason why escalated conflicts are so hard to undo is that when more aggressive tactics are used by one side, they are often mirrored by the other side, producing a vicious cycle", 
which they liken to the cold war.

My previous research has demonstrated that there is a significant difference in the way cultures indicate and manage conflict (Stadler, 2007; 2013) and as such, even more care has to be taken. Hence, it is imperative to gain a better understanding of the workings of discursive conflict markers in intercultural business interactions and transactions.

Relationship conflict can be triggered by virtually anything in intercultural settings. Ranging from inadequate hotel selection, mismanaged hosting practices, inappropriate seating arrangements (cf. Spencer-Oatey and Xiong, 2008), to non-verbal expressions such as staring or pointing (cf. e.g. Stadler, 2007) or simply a lack of verbal response and inclusion, i.e. overlooking a contribution (see example below). Wall and Callister (1995) provide an extensive list of conflict causes, including individual characteristics (personality, values, goals, commitment to position, stress, anger, desire for autonomy), interpersonal factors, such as perceptual interface (perception that other has high goals, other's intentions counter to party's, other's intentions counter to party's fairness norms, other's behavior seen as harmful, distrust), communications (distortions and misunderstandings, hostility, dislikes, insults), behaviour (blocking party's goals, power struggles), structure (closeness, power imbalances, interdependence, status gaps, preferential treatment of one side), and previous interactions (past failures to reach agreement, history of conflict, locked-in conflict behaviours) (for a complete list refer to Wall and Callister 1995: 518).

While theirs is a list of more concrete and tangible aspects of conflict, working somewhat on the premise of mal-meanings and negative prior dispositions, my own research has uncovered that many conflict triggers, in fact, constitute seemingly innocuous actions, with both parties initially approaching the meeting well-meaning and with positive intentions. However, even very small mishaps can have detrimental effects equivalent to a proverbial slap in the face. Much of what can constitute conflict triggers is non-verbal and fairly subtle. Therefore it is paramount to the success of such meetings to attune to the speaker's behaviours and respond to their early indications and warning signs efficiently.

\section{Methodology}

The focus of this paper is on interactional relationship conflict and its consequences. With communication as its platform, I employ a discourse analysis approach that takes an in-depth micro-level look at interactional structures, markers and indicators that signal early stages of potential interactional problems. While there are multiple approaches to text analysis, I chose to focus on discourse analysis, as it approaches interaction from an angle that represents culture and society and focuses on the individual, as well as on interpersonal, relational processes. Unlike the notion of monologic communication, which sees meaning as imposed, Bakhtin's notion of dialogic communication sees meaning as an interactional process (Klages, 2012). Discourse analysis, likewise, sees meaning as a negotiation process between interactional parties. Instead of a singular truth, interactants work together to construct a shared truth, which, however, is always somewhat subjective and is never completely and fully aligned between the individual speakers. The idea behind this approach is context-based co-construction of meaning instead of an essentialist truth. However, in addition to the notion of dialogic communication discourse analysis further draws on the larger notion of pragmatic meaning making and meaning negotiation as an interactional process with a strong reliance on social, situational and interactional context cues (cf. Gumperz, 1982, 1992), on which interactants rely to interpret communication. Rather than regarding interaction as a vacuum, discourse analysis sees the social, situational and contextual embedding of an interaction as essential for deriving meaning, as the interlocutors themselves rely on these cues in order to give meaning to the words that are spoken. This means both, a multimodal approach (not only verbal communication, but also prosodic (vocal) and non-verbal cues) to the spoken language and a larger awareness of the communication setting and the socio-pragmatic factors (e.g. power, distance, and imposition; cf. Brown \& Levinson, 1987) within which frame the interaction takes place.

Phillips et al. (2004, p.635) hold that linguistic processes constitute institutional reality; as such, they argue that discourse analysis provides a coherent framework for an investigation into institutional practices. Indeed, discourse analysis as a methodological approach for the investigation of both intercultural interactions and business communication is becoming increasingly popular. Discourse is a regulated way of speaking that is defined by culture (both national and institutional culture). In order to investigate intercultural effectiveness, there is thus no better way than to look into the intricate workings of discourse in detail. Barker and Galasinski (2001, p.24) argue that other approaches and types of analyses to cultural studies will be "enriched through the benefits of detailed linguistic insights". In a similar vein, Van de Mieroop and Vrolix (2014, p.160) state that discourse analysis enables researchers to look carefully at action interactional practices there therefore "enables the researcher to provide valuable insights in how everyday workplace activities are accomplished".

More than the mere production of speech, discourse analysis allows us to explore not only speech production, but also how messages are perceived, received and responded too. In order to determine the value of attuning to conflictual stances intercultural communication, it is essential to identify if and how interactants themselves benefit during the interaction and their reactions to attentive listenership (or the lack thereof) serve as vital indicators for the successful application of attuning in action. 
This approach stands in contrast to other forms of text analysis, such as narrative analysis that focuses exclusively on personal meaning-making, interactional sociolinguistics, which focuses on imposing and managing frames of meaning and identities, conversational analysis, which focuses on accomplishing interactional life in real time, discursive psychology, which focuses on displaying and deploying psychological states and critical discourse analysis, which focuses on constituting and regulating the social and political world, according to Antaki (2008). Although psychological processes and power structures, arguably, impact all types of interactions, this paper focuses solely on interpersonal effects derived from interactional processes that have conflict at their core.

In this paper then, I will demonstrate on the basis of interactional examples that, where conflict markers are identified in the early stages and actions are taken accordingly, misunderstandings and conflict can be avoided. However, where such precautions are not observed or interactants fail to respond in a timely and adequate manner, business meetings can come to a complete breakdown as a consequence of the resulting conflict.

\section{Data}

The data consists of a collection of a wide variety of business meetings from a range of business sectors across various cultural contexts, including China, Japan, Singapore, Bali, Britain and Germany as well as a variety of interactional and transactional intercultural meetings collected in China, Japan, Singapore, and Germany, with participants from North America, South America, Europe,

\section{Asia and Oceania.}

The excerpts presented from this data discuss in a fair bit of detail how communicational misunderstandings, disagreements and misalignments occur and demonstrate both cases for successful conflict avoidance as well as examples of escalation and their undesirable consequences. Consequences are not merely assessed on the basis of the communication excerpts, but also rely on the feedback given by the interactants themselves in follow-up interviews, in which the negative effects of these communication issues become very clear. A combination of ad-hoc speech and post-hoc assessment provides critical insights into both interactional processes as well as the consequences of conflict on interpersonal relationships.

\section{Successful Attuning}

The following excerpt takes place between one British participant (speaker B) and one Chinese participant (speaker $\mathrm{C}$ ). Prior to the following excerpt speaker $\mathrm{C}$ outlines to speaker B what he sees as key differences between British and Chinese educational systems. He mentions the impact of cultural differences in terms of learning style and teaching methods. A concern seems to him that more research needs to be carried out into differences in learning styles, as Chinese students rely more heavily on their teachers and learning is more controlled by teachers. As a consequence he cautions that the compatibility of the two cultural systems may be compromised. The British colleague then initiates the subsequent clarification sequence.

\section{Excerpt 1: Being clear}

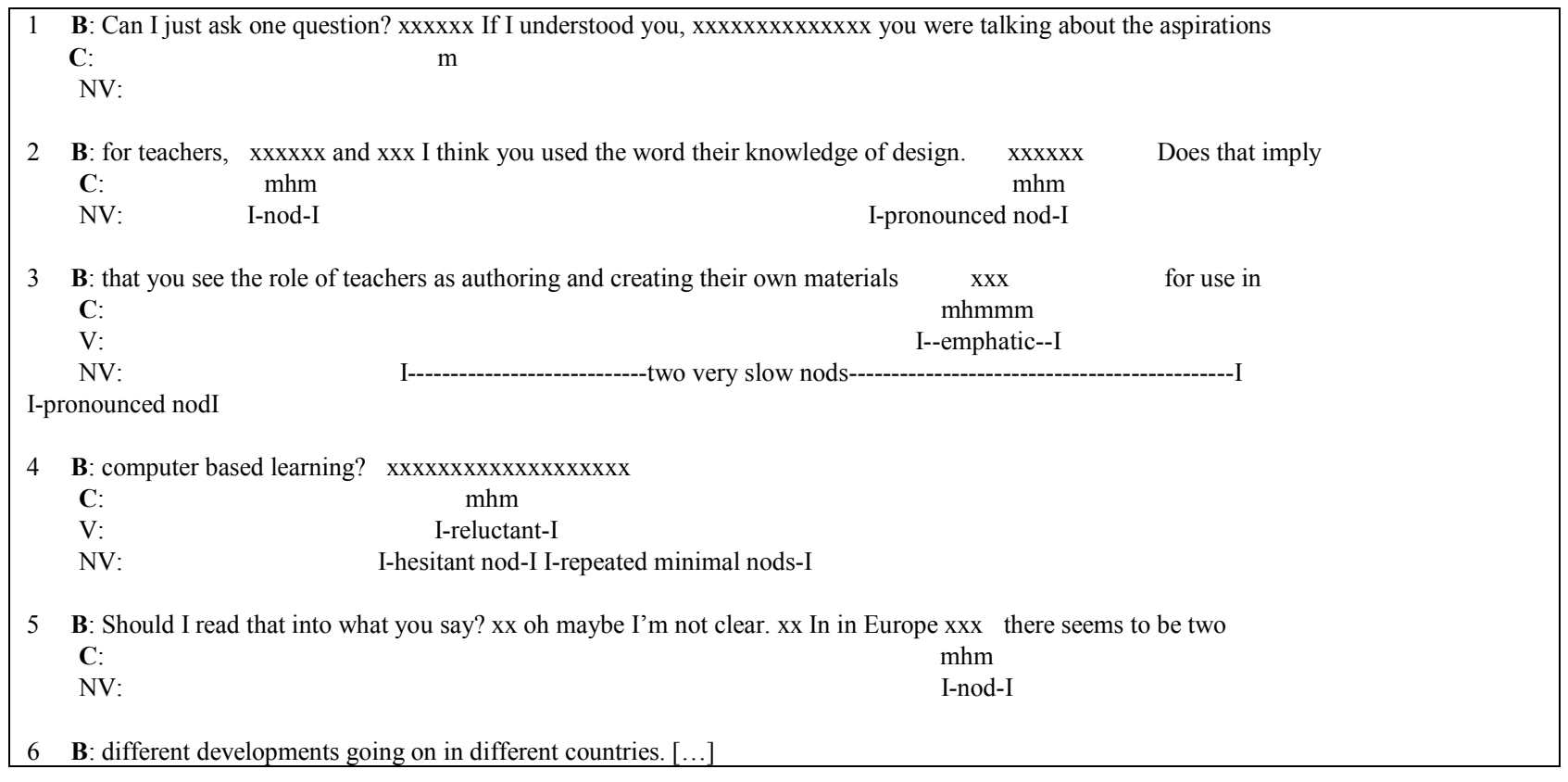


Speaker B demonstrates unmistakably that he has been listening actively to speaker C's prior monologue. He signals his intentions clearly with statements such as 'can I ask just one question' and 'if I understood you'. Seeing as speaker $\mathrm{C}$ is not responsive to his request for clarification speaker B follows this up with another question 'should I read that into what you say' indicating to $\mathrm{C}$ once more what exactly he is asking of speaker $\mathrm{C}$. Then, without leaving any pause and without leaving time for speaker $\mathrm{C}$ to respond, speaker B immediately concludes that he has not been understood. This prompt reaction of speaker B clearly indicates that he has been clearly attuned to his interlocutor. $\mathrm{He}$ could not possibly have come to this immediate conclusion if he had not been paying attention and oriented to speaker C's vocal and non-verbal feedback. While backchanneling in intercultural interactions is a common phenomenon to signal that the listener is attentive and to encourage the speaker to carry on, in intercultural communication backchannels carry more weight. Speaker C employs them to signal not only his attention, but also his understanding (or a lack thereof). He does so by responding with a backchannel after every single turn-final intonation pattern that speaker B produces up to this point (except in line 1 after 'if I understood you'). He produces this feedback with varying degrees of emphasis. However, speaker $\mathrm{C}$ gives clear verbal, vocal and non-verbal responses throughout speaker B's request for clarification, at the relevant point, that is after speaker B finishes asking for clarification in line 4, there is a crucial shift in speaker C's feedback. While he still produces the same backchannels, at this critical point in the interaction they lack in emphasis and prominence, the additional hesitant vocal quality add to the hesitant non-verbal cue and indicate a clear lack of understanding. While speaker $\mathrm{C}$ does not at any point verbalize his lack of understanding, he nevertheless communicates this clearly as part of his role as addressee by providing feedback during speaker B's turn. Speaker B clearly orients to this, not only because he launches a further attempt to make the request for clarification a successful one, but also by orienting to the lack of any form of feedback after he restates his request for clarification in line 5 'should I read that into what you say'. He does not need to wait for a verbal response from speaker $\mathrm{C}$, because he had been attuned to speaker C's hesitancy and the subsequent lack of backchannels, which allows him to conclude instantaneously that he has not been fully understood. For full discussion refer to Stadler (2013). This form of attention to the interactional partner and their hesitations (and thus concerns) avoids leaving negative impressions on the interactional partners and therefore avoids friction and conflict.

\section{Failure to Attune}

In the following excerpts, on the other hand, it becomes clear that interactional partners are not always attuned and that this sometimes leads to dire consequences.

In excerpt 2 , two of the interactants have been with the company for a number of years, while one of the interactants recently joined the team. The new team member, Fay, is a Filipina. The slightly more senior local team member, Sue, is Singaporean and the HR person who constitutes part of this meeting, $\mathrm{Al}$, is Australian. The purpose of the meeting is to discuss how to move an existing project (a training session for junior staff) further and also to discuss Fay's involvement in the project.

\section{Excerpt 2 The Video}

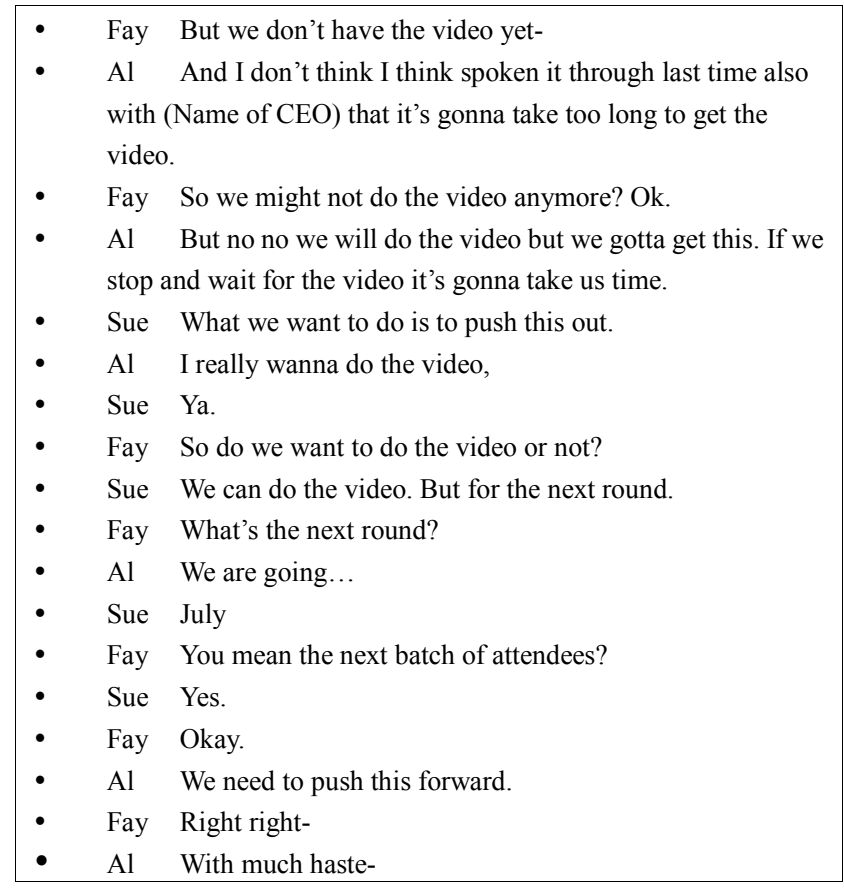

Excerpt 3 No Time

- Al Here here. I have done a lot of work here already and its... its

Sue I'm trying let's not reinvent wheels urm because I really want to push this out and that's what John is- I'm getting very impatient because I've been talking about this for months.

- $\quad$ Al For months. And we are coming back and it looks to me like there is a significant amount of rewrite that you are proposing.

- Sue Yes.

- Fay It's...

- $\quad$ Al And who's gonna do it? Cos I'm not. Cos I've spent my time- a lot of time on this already and I'm not going back and rewrite it.

- $\quad$ Fay Okay...so-

- $\mathrm{Al}$ And i've done a lot of thinking about the structure of the exercises and everything so... 
Excerpt 4 Client Brief

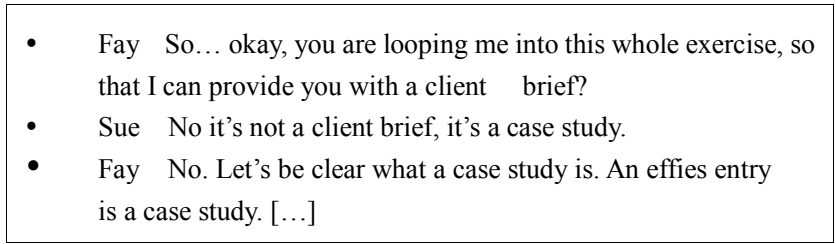

Excerpt 5 We're All Busy

- $\quad$ Fay Ok, just give me the brief. What do you want me to do? Ok basically we are all busy people right? We are all doing all sorts of stuff, you guys are not the only busy people in this office. And I also put in time for this so can you just tell me now very clearly, because we are talking about giving instructions or briefing people, so what exactly in black and white do you need me to work on. What sort of inputs do you want.

In excerpts 2-5 an increasing escalation of disagreement, dissatisfaction, conflict and anger can be observed, which stems from a lack of attuning to the warning signs of pragmatic markers employed. Throughout the progression of this increasingly heated exchange, Fay uses multiple forms of markers. At first, she relies on repetition, bringing the topic back to the video 3 times over an exchange of only a few lines. While $\mathrm{Al}$ and Sue are very reluctant to provide a clear answer, Fay obviously pushes the topic and this unwillingness to let this issue go clearly indicates conversational problems. She also uses multiple verbal markers, especially 'but' and towards the end when the interactants are falling out the much stronger marker 'no'. However, characteristic for her use is the recurrent use of markers 'so', 'okay' and 'right'. While these do not seem like markers of conflict initially, the prosody with which these cues are produced betray her irritation. The increasingly stroppy quality of tone in her voice as well as the curt pronunciation of 'so', the rush-through of 'ok' and the prolonged and level trail off of 'right' signal that none of these are verbal fillers (i.e. place holder like the Japanese forms 'etto' or 'ano') of forms of genuine agreement. Instead, they are indicators of irritation and tension that keep building up to conflict until the interaction breaks down entirely immediately subsequent to excerpt 5 .

In the following excerpt another form of lack of attuning can be observed, which constitutes less in the interactants taking up verbal reprises to someone's suggestions and actions, but rather a complete lack of attention to someone's contributions. While this excerpt does not stem from a business setting and the discussion of where to eat lunch hardly has serious implications for anyone, it is nevertheless obvious that such inattention to someone's contributions and wishes is not conducive to interpersonal relations, which cement business deals in many countries.

As can be seen, it is certainly not for a lack of trying on Ken's part that his contributions are not taken up. Despite his multiple repetitions however, his contributions are completely ignored by all the other participants. While this may seem rather innocent, it became clear in the subsequent short interviews and feedback questionnaires that Ken harboured resentment regarding this inattention to his conversational efforts and gave fairly negative feedback on the behaviours of the other participants even though he considers them to be friends.

Excerpt 6 Noodles or Rice

\begin{tabular}{|c|c|c|c|}
\hline 1 & & Olle & It's up to you Malte. $>$ You decide now. $<$ \\
\hline 2 & & Malte & I decide? \\
\hline 3 & & Nadja & [Ya.] \\
\hline 4 & & Monika & [Ya.] \\
\hline 5 & & Olle & [Ya.] Where to eat. \\
\hline 6 & & Nadja & Ya. \\
\hline 7 & $\rightarrow$ & Ken & Noodles or [rice. ] \\
\hline 8 & & Nadja & [What] to- \\
\hline 9 & & Monika & (It goes) by the [maj]ority.] \\
\hline 10 & & Malte & [Poo] \\
\hline 11 & & Olle & [Poo.][Awesome ] \\
\hline 12 & & Nadja & [Poo? Poo?] \\
\hline 13 & & Monika & Poo? \\
\hline 14 & & Olle & Free poo- ((high pitched) $)$ \\
\hline 15 & & Ingrid & What's funny about that. \\
\hline 16 & $\rightarrow$ & Ken & Noodles or rice. \\
\hline 17 & & Nadja & Poo? No:. ((facing Malte)) \\
\hline 18 & $\rightarrow$ & Ken & What do you think. Noodles or rice. \\
\hline 19 & & Ingrid & En:::: \\
\hline 20 & & Olle & Poo? \\
\hline 21 & & Nadja & noodles. ((facing Ken $))$ \\
\hline 22 & & Monika & (sushi) \\
\hline 23 & & Ingrid & I don't want sushi. \\
\hline 24 & & Olle & You don't want (a shoe)? \\
\hline 25 & & Ingrid & In that case, \\
\hline 26 & $\rightarrow$ & Ken & Wok. \\
\hline 27 & & Ingrid & Ok; right. \\
\hline 28 & & Malte & (???) garden then- \\
\hline 29 & & Monika & No. \\
\hline 30 & & Olle & Yeah; \\
\hline 31 & & Nadja & [No::.:::.::] \\
\hline 32 & & Ingrid & [YEAH::] \\
\hline
\end{tabular}

What can be concluded from these excerpts is a) that conflict can be triggered by various and often very subtle means, not always deliberate action, but often the inaction or overlooking of meaningful conversational means, b) that innocuous conversational actions can lead to dire consequences and full-blown conflict, and c) that such conflict scenarios can be avoided if early action is taken through attuning.

\section{Conflict Markers}

Conflict markers can be both cultural and idiosyncratic to some extent, seeing as human beings are all individuals and have independent communication styles to some extent. However, while it is important for interactional partners to attune to the specific signals of each of their interactional partners, there are some markers that indicate potential upcoming friction, dissent and conflict span across the various contexts of my data. 


\begin{tabular}{|c|c|}
\hline \multicolumn{2}{|r|}{ Conflict Markers } \\
\hline Verbal & $\begin{array}{ll}\text { - } & \text { Repetition } \\
\text { - } & \text { Reset/repair } \\
\text { - } & \text { Stuttering } \\
\text { - } & \text { Fillers (e.g. uh, uhm as delay mechanism) } \\
\text { - } & \text { Pragmatic markers (e.g. no, but, so, initial } \\
& \text { agreement + disagreement marker) }\end{array}$ \\
\hline Non-verbal & $\begin{array}{ll}\text { - } & \text { Withholding of non-verbal backchannels (i.e. } \\
\text { - } & \text { listener feedback) } \\
\text { - } & \text { Facial expressions (e.g. raised eyebrows) } \\
\end{array}$ \\
\hline Prosodic & $\begin{array}{ll}\text { - } & \text { Withholding of prosodic backchannels } \\
\text { - } & \text { Change in intonation contour (e.g. mid-level to } \\
\text { - } & \text { Calling) } \\
\text { - } & \text { Change in pitch } \\
\text { - } & \text { Pauses in speech rate (i.e. tempo) } \\
\text { - } & \text { Hesitation } \\
\text { - } & \text { Delay }\end{array}$ \\
\hline
\end{tabular}

Although this list is by no means exclusive, the above table represents a list of frequently observable items that indicate interactional trouble. It is somewhat difficult to capture accurately the exact nature of gestures that mark conflict, as it is a matter of nuances and idiosyncratic movements and habits. In having said this, no matter what an individual's use of gestures is, a sudden change in the frequency or quality of gestures is a strong indicator. In excerpt 1 , it becomes abundantly clear that withholding is the strongest indicator for interactional trouble.

Verbal features and prosodic markers are far more consistent. Verbally, repetition is a strong sign that an interactant is unhappy with how the conversation is progressing, if someone repeatedly returns to the same topic and issue, then it is certain that a lack of clarity prevails or a lack of satisfaction with interactional outcomes underlies such speech choices. Clear indicators for disagreement consist of verbal markers such as outright 'no' (which is absent in some data sets, especially from Asian contexts, where such a direct form of dissent is rarely used), but also more 'subtle' markers that achieve their effect of indicating dissent through their habitual usage, especially 'but' and 'well' in the English language. Commonly these are combined with 'yes', 'yeah', 'ok' as a pre-face to indicate initial agreement. It is therefore important to pay close attention to the quality of a 'yes' in order to identify early whether it is an indicator actual agreement or an early warning sign for upcoming dissent and marks only a form of what is commonly referred to as 'token agreement' (Stadler, 2007). Similar observations can be made for markers such as 'so', 'right', 'okay', which do not clearly mark dissent, but are often observable in a context of dissent. Throughout excerpts 2-5, Fay makes extensive use of these markers and they are in no way meant to indicate actual agreement. A sensitivity towards these markers can help with communicational trouble-shooting, before a business meeting escalates, as is the case in this particular meeting that results in Fay losing her temper and a complete breakdown and abrupt ending of the meeting immediately after excerpt 5 in which Fay takes a very stroppy stance towards her more senior colleagues.

However, I would argue that the most telling indicator of all is the use of prosodic nuances and subtle changes to a speaker's habitual speech prosody. This is in part due to the fact that people rely on prosody more than any other communicative channel to give meaning to an utterance (cf. e.g. Stadler, 2007) and partly due to the fact that even if people want to avoid showing their attitudes towards speech, it is nearly impossible to suppress emotions and attitudes from speech prosody (while it is fairly easy for people to refrain from showing such attitudes through verbal and non-verbal means). Therefore, a lot more truth rings in how we say things rather than in what we say. Mehrabian (1981) infamously equated the display of emotions as $7 \%$ verbal + $38 \%$ vocal $+55 \%$ facial. However, according to Tanaka et al. (2010), this very much depends on a speaker's cultural background. Japanese people, they argue, habitually "control the display of their own feelings in the face", and therefore rely more on voice than facial expression in their observation in others (p. 1262). This is true for many Asian cultures, hence, a strong focus on prosodic markers is particularly important in an Asian context, though it is critically important to the detection of conflictual stances and attitudes anywhere.

\section{Concluding Remarks}

As this paper demonstrated, interaction and relational conflict, which invariably involve some form of attitudinal involvement, ranging from irritation and dissatisfaction to anger, can be identified in its early stages. By paying particularly close attention to verbal markers, non-verbal cues as well as speech prosody and can thereby serve as preventative means to avoid conflict from arising at a later point. The competency of attuning into an interlocutor's idiosyncratic habits constitutes a vital tool for conflict management in intercultural communication in general and international business discourse in particular. To this end, interlocutors need to be able to tune into a speaker's habitual speech practices in order to identify 'unusual' or 'abnormal' patterns in a particular speaker's utterances.

\section{REFERENCES}

[1] Antaki, C. (2008). Discourse analysis and conversation analysis. In P. Alasuutari, L. Bickman \&

J. Brannan (eds.), The Sage Handbook of Social Research Methods (pp. 431-446). London: Sage.

[2] Barker, C. \& Galasinski, D. (2001). Cultural Studies and 
Discourse Analysis. London: Sage.

[3] Brown, P. and Levinson, S. (1987). Politeness: Some Universals in Language Usage. Cambridge: Cambridge University Press.

[4] DeDreu, C.K.W. \& Weingart, L.R. (2003). Task versus relationship conflict, team performance, and team member satisfaction: A meta analysis. Journal of Applied Psychology 88(4), 741-749.

[5] Elmer, D. (1993). Cross-Cultural Conflict. Downers Grove, IL: InterVarsityPress.

[6] Friedman, R.A. and Currall, S.C. (2003). Conflict escalation: Dispute exacerbating elements of e-mail communication. Human Relations 56(11), 1325-1347.

[7] Gumperz, J.J. (1982). Discourse Strategies. Cambridge: Cambridge University Press.

[8] Gumperz, J.J. (1992). 'Contextualization and understanding'. In A. Duranti \& C. Goodwin (eds.), Rethinking Context (pp. 229-252). Cambridge: Cambridge University Press.

[9] Klages, M. (2012). Key terms in literary theory. London: Continuum.

[10] Mehrabian, A. (1981). Silent messages: Implicit communication of emotions and attitudes. Belmont, CA: Wadsworth

[11] Phillips, N., Lawrence, T.B. \& Hardy, C. (2004). Discourse and institutions. Academy of Management Review 29(4), 635-652.

[12] Simons, T. L., \& Peterson, R. S. (2000). Task conflict and relationship conflict in top management teams: The pivotal role of intragroup trust. Retrieved [31.05.2016], from Cornell University, School of Hospitality Administration: http://scholarship.sha.cornell.edu/articles/719
[13] Spencer-Oatey, H. \& Xing, J. (2008). Issues of face in a Chinese business visit to Britain. In H. Spencer-Oatey (ed.), Culturally Speaking (pp. 258-273). London: Continuum.

[14] Stadler, S. (2013). Why Intercultural Interaction Demands a Dual Role-Relationship. Language and Dialogue, 3 (2), 167-185.

[15] Stadler, S. (2013). Cultural Differences in the Orientation to Disagreement and Conflict. China Media Research; Special Issue: Managing Language and Cultural Challenges in Cross-Border Deal-Making 9 (4), 66-75.

[16] Stadler, S. (2011). Intercultural Competence and its Complementary Role in Language Education. In Perez-Llantada, C. and Watson, M. (eds.), Specialized Languages in the Global Village: A Multi-Perspective Approach. Cambridge: Cambridge Scholar Press, 259-284.

[17] Stadler, Stefanie. (2007). Multimodal (Im)politeness: The verbal, prosodic and non-verbal realization of disagreement in German and New Zealand English. Hamburg: Verlag Dr. Kovač.

[18] Tanaka, A., Koizumi, A., Imai, H., Hiramatsu, S., Hiramoto, E. \& de Gelder, B. (2010). I feel your voice: Cultural differences in the multisensory perception of emotion. Psychological Science 21(9), 1259-1262.

[19] Van de Mieroop, D. and Vrolix, E. (2014). A discourse analytical perspective on the professionalization of the performance appraisal interview. International Journal of Business Communication 51(2), 159-182.

[20] Von Glinow, M., Shapiro, D.L, \& Brett, J.M. (2004). Can we talk, and should we? Managing emotional conflict in multicultural teams. Academic Management Review 29, 57892.

[21] Wall, J.A. and Callister, R.R. (1995). Conflict and its management. Journal of Management 21(3), 515-558. 